\title{
Air pollution and emigration behaviors - evidence of Hanoi
}

\author{
Authors: Loc Quang Nguyen*, Ananya Singh ${ }^{1}$, Saanvi Jain ${ }^{2}$, Khangai Shirchin ${ }^{3}$ \\ SP Jain School of Global Management, New South Wales 2141, Australia \\ *Corresponding author: loc.bs21don038@spjain.org \\ ${ }^{1}:$ ananya.bs21dmu013@spjain.org \\ 2: saanvi.bs21dmu005@spjain.org \\ 3:khangai.bs21don023@spjain.org
}

(Un-peer-reviewed manuscript v1)

December $16^{\text {th }}, 2021$

\begin{abstract}
Expeditious increase in population and industrialization has led to alarming rates of air pollution in all countries. However, developing economies have had to face a more adverse and severe impact. This had led to many changes in the day to day living of citizens. In this paper we have focused on the psychological process and predictors of migration intention of the people living in Hanoi, Vietnam. Two stratified random datasets of 475 people were used, and Bayesian analysis was performed on this dataset. We found out that the intent to move was negatively associated to the individual's satisfaction with air quality. We also found that people who have family members that have fallen victim to a disease caused by air pollution are more likely to migrate. This paper discusses an important topic: immigration of the younger demographic, i.e. the Hanoi workforce, which may cause restrictions and hurdles in the city's urbanisation and development. The findings suggest that, if measures against air pollution are not taken, economic forces may be disrupted, posing a threat to urban growth. As a result, collaborative activities and steps need to be taken by the government to curb this unfortunate consequence.
\end{abstract}

Keywords: Air pollution, emigration, Vietnam

Introduction

Air pollution is all around us, and poses as a complex socio-environmental problem. The relationship between health and air pollution as a subject has been researched intensely for the last few decades. An increase in mortality, physiological stress, bioaccumulation and disturbance in activities of certain wildlife species has been observed with an increase in pollution [1]. In addition, older age groups are at a higher risk of air pollution related mortality [2]. More than 25,000 new cases of chronic bronchitis in adults and more than 290,000 episodes of bronchitis in children are recorded per year as a result of exposure to polluted air [3]. This health crisis is predominantly a problem in developing countries; $65 \%$ in Asia alone [4]. 
In Vietnam, after Đổi Mó́i and urbanisation- there was a boom in the population, and soon transportation, power plants, industry and fuel usage became major contributors to air pollution. The current study's purpose is to understand the intention behind the out-migration of the people of Hanoi - one of the most polluted capital cities [5].

Migration is a complicated process in which a number of 'macro', 'meso', and 'micro' elements interact to influence an individual's decision to migrate. Elements such as political, demographic, socioeconomic, and environmental conditions are macro elements, these are out of an individuals' control and often force migration unto them. Meso elements are influenced by social life and relationships. However, 'micro-factors' such as education, religion, marital status, and personal attitudes about migration play a significant impact in determining whether to migrate.[6]

Luong et al. found that the increase in particulate matter was positively related to the respiratory admissions of young children in hospitals of Hanoi [6]. If the environmental conditions are not improved in the city of Hanoi in coming years, then it will lead to migration of young, healthy people, and lead to a shift in the economic centre of the country hindering urban development.

There have been many studies carried out to find out the impact of air pollution on the lives of people in the urban cities [7,8], which inspire to conduct this study. This study is a part of assignment project of Introduction to Statistics and Probabilities class, which attempts to enlighten up the fact as to why the local government should take steps to improve the air quality and also verifies the effects of population migration caused by air pollution.

The objective of this study is to:

- To examine the impact of satisfaction, health condition being affected due to current air quality, and moving cost of migration on the intent to migrate.

Materials and method

Materials

The information used in this study came from two available databases about Hanoi residents' attitudes toward air pollution [9, 10]. The two datasets have been procured from two different survey collections using stratified random sampling methods at the central and suburban areas of the city, respectively. The survey collections were organized in November and December of 2019. Hanoi was chosen as the study site for the following three reasons:

1) Hanoi was ranked among the top 10 of the most polluted capital cities around the world [5];

2) Hanoi is one of the fastest growing cities in Vietnam;

3) Hanoi is the second largest and most populous city in Vietnam.

According to Khuc et al. [10], the survey collection comprised of three steps. To begin, the collectors were hired and well compensated in order to motivate them to perform well during the collection procedure. Two four-hour lectures were also arranged to help the collectors comprehend the project's goals and the questionnaire. During the event, participants will learn the necessary skills and procedures for extracting information from respondents.

Two pilot tests showed that the final version was error-free, clear, and easy to grasp. Finally, the collectors conducted face-to-face interviews with the respondents and maintained mutual engagement and communication throughout the survey collection process to resolve any concerns or queries that arose. The variables description is presented in Table 1: 
Table 1: Variable's description

\begin{tabular}{|c|c|c|c|}
\hline Variable & Meaning & Type of variable & Value \\
\hline MoveCity & $\begin{array}{c}\text { Whether the } \\
\text { respondent had the } \\
\text { intention to out- } \\
\text { migrate from Hanoi } \\
\text { due to air pollution }\end{array}$ & Binary & Yes $=1$ \\
\hline AirSatisfaction & $\begin{array}{c}\text { The respondent's } \\
\text { evaluation towards } \\
\text { the current air quality }\end{array}$ & Continuous & $\begin{array}{c}\text { From 1 (very } \\
\text { dissatisfied) to 5 } \\
\text { (very satisfied) }\end{array}$ \\
\hline AirDisease & $\begin{array}{c}\text { Perceiving a family } \\
\text { member has been } \\
\text { sicked because of air } \\
\text { pollution }\end{array}$ & Binary & $\begin{array}{c}\text { No/ Do not know }=0 \\
\text { Yes }=1\end{array}$ \\
\hline
\end{tabular}

From the dataset, we created two variables for statistical analysis: one predictor variable and one predicted variable. The predicted variable MoveCity is made up of two 'yes' and 'no' responses to the question "Do you intend to transfer your family and work in another province with less pollution?" The AirSatisfaction variable measures how satisfied urban residents are with the present air quality level by asking the question "Overall, how satisfied are you with the air quality?" Besides the variables in the dataset, we generate one variable named AirSatisfaction*AirDisease which is the interaction between AirDisease and AirSatisfaction.

\section{Model construction}

We build a model based on the Mindsponge Framework of information processing to examine the behaviour of domestic migration driven by air pollution [11,12]. The paradigm has been used successfully to investigate the psychological factors that govern human behaviour [13-15]. The goal of the model is to look at the effects of people's contentment with present air quality as well as the moderation effects of air pollution on their families' health. In the Mindsponge framework, the mind is compared to a sponge that squeezes out unsuitable values while absorbing new ones that are compatible with its core value. These core values can be governed by financial benefits or comfortable living.

For ideation to develop within an individual's mind, the Mindsponge mechanism implies that information must pass through a multi-filtering system employing cost-benefit assessments. When the idea of migration is accepted into an individual's brain as a good choice, we anticipate that they will intend to move and work in another city. Migration, on the other hand, will be rejected if it is deemed to be unfavourable, and the individual will have no desire to migrate.

Based on this premise, we hypothesised that someone who is more satisfied with existing air quality views the act of relocating to another place as less advantageous. Furthermore, we suspected the presence of two additional elements that influence the cost-benefit analysis of the migration notion by moderating the impact of air quality satisfaction on migration intention, 
namely, disease caused by air pollution. As a result, we theorized that people are more likely to migrate if they observe their relatives' health is deteriorating.

Model can be presented by the following specification:

Model: MoveCity $\sim a_{-}$MoveCity + b_AirSatisfaction_MoveCity * AirSatisfaction + b_AirDisease_AirSatisfaction_MoveCity * AirDisease *AirSatisfaction

Methods and validation

The Bayesian analysis was employed because of the following mentioned five reasons. Firstly, It is important to note that science is facing a reproducibility crisis right now, meaning a lot of studies across different fields (psychology [16] and social sciences [17] are not excluded) could not be replicated. One of the main reasons is suggested to be the wide sample-to-sample variability in the $p$-value. For not being dependent on the $p$-value, the Bayesian inference approach is a good alternative because it treats all the properties probabilistically, including the unknown parameters. Secondly, this characteristic of Bayesian analysis has high compatibility with the current study's design, which is explanatory research. The study employed the Mindsponge framework to explain the psychological process that might lead to the migration intention. Considering the above mentioned reasons we can say that the Bayesian analysis helps us consider impacts of undetermined factors while maintaining the rule of of parsimony for the explanation [18].

The impact of AirDisease variables was investigated in our models. Regressing the supposedly correlational variable could lead to confusing results. As a result, we decided to consider the variable as an interaction variable in order to make its impact non-linear. The treatment complicated the model, necessitating a larger sample size for accurate estimation [19]. Nonetheless, the Markov Chain Monte Carlo approach, which incorporates Bayesian analysis, creates a huge number of parameter samples using stochastic Markov chain processes, which aids in the accurate fitting of complex models. In addition, MCMC Bayesian analysis delivers a more precise small sample size dataset than the Frequentist approach [20].

For validating the simulated posterior outcomes, we adopt a three-pronged validation strategy. Initially, we tested with the convergence check using both the diagnostic statistics and plots. The statistics include the effective sample size, and the Gelman shrink factor, while the plots include the trace plot, Gelman plot, and an autocorrelation plot. The Results section contains a more detailed description and interpretation of the diagnostic statistics, plots, weight comparison, and prior-tweaking technique.

The bayesvl R package was used to perform Bayesian analysis in the current study for three reasons: 1) it is a cost-effective alternative; 2) it has a great capability to visualize eye-catching graphics; and 3 ) it is easy to be operated $[21,22]$.

\section{Results}

We examined the Model using a Bayesian analysis to test the assumptions we made before. We used Bayesian MCMC simulation with 5,000 iterations, 2,000 warm-up iterations, and four Markov chains to estimate these three models. All of the models' simulated results and technical validity using 'uninformative' priors are given in this way. The analysis was carried out on samples that had a higher proportion of male respondents than female respondents $(54.53$ percent versus 
45.26 percent). The majority of the samples were from people aged 10 to 30 . (61.47 percent). Due to air pollution, nearly $5 \%$ of the 475 respondents said they planned to relocate their family and employment in another city.

Through these graphs and models we found out that air satisfaction and air disease both have a direct relation to move city, air satisfaction has an inverse relation and air disease has a direct one.

The model examined the effects of citizens' satisfaction with air quality and its interactions with perceived air quality affecting their relatives' health conditions on migration intention. The model's logical connection is shown in Figure 1.

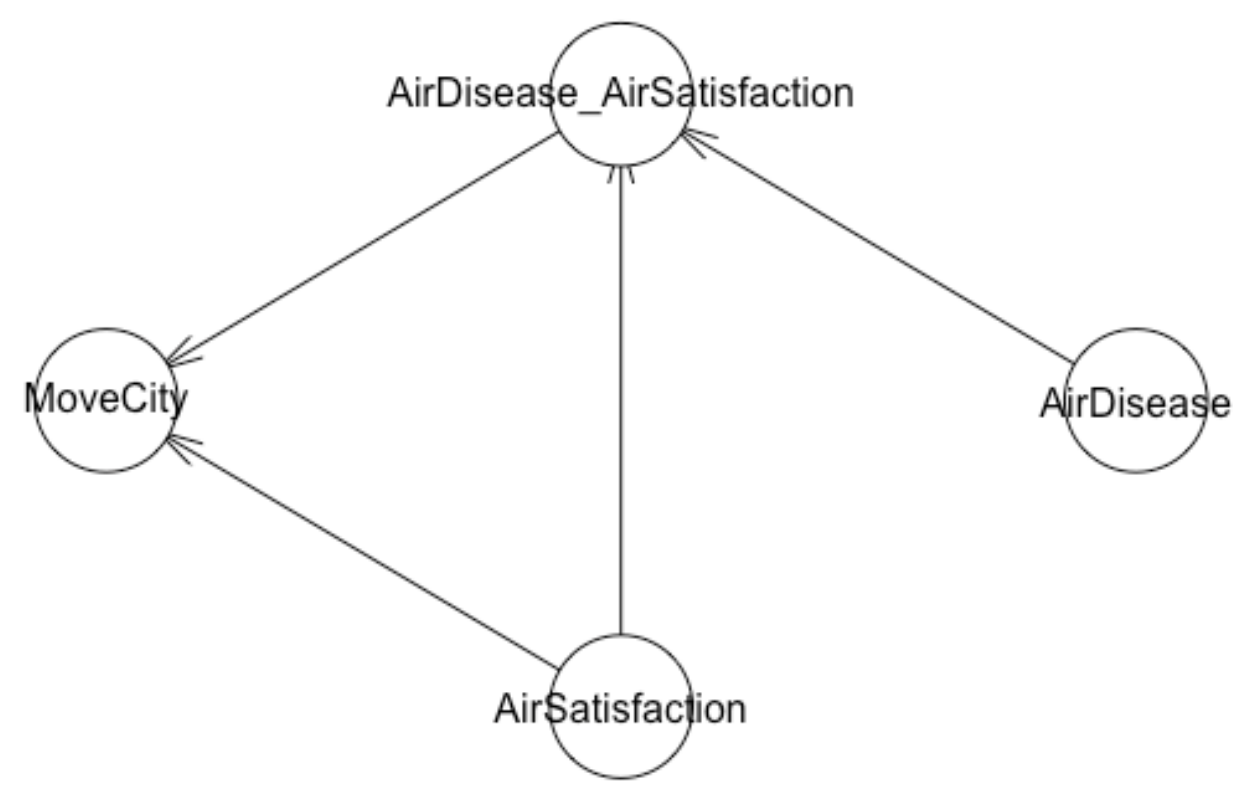

Figure 1: Model's logical network

The effective sample size ( $n \_$eff $\left.>1000\right)$ and Gelman shrink factor $($ Rhat $=1)$ of all simulated posterior coefficients portray a good convergence of the model's Markov chains (see Table 2). The convergence is also visually diagnosed using the trace plots, autocorrelation plots, and Gelman plots.

Table 2: Simulated posterior coefficients for Model 


\begin{tabular}{|l|l|l|l|l|}
\hline Parameters & Mean & SD & n_eff & Rhat \\
\hline Constant & -1.95 & 0.72 & 4252 & 1 \\
\hline AirSatisfaction & -0.18 & 0.33 & 4045 & 1 \\
\hline AirSatisfaction*AirDisease & -0.21 & 0.20 & 5672 & 1 \\
\hline $\begin{array}{l}\text { Note: } \\
* \text { SD S Standard deviation } \\
* * \text { The effective sample size (n_eff) and Gelman value (Rhat) of simulated results with different } \\
\text { priors are almost similar, so only the n_eff and Rhat of simulated results using uninformative } \\
\text { priors are presented. }\end{array}$ \\
\hline
\end{tabular}

The trace plots of all posterior parameters are shown in Figure 3. The posterior values of each parameter are represented on the $y$-axis of the trace plot, while the simulation iteration order is represented on the x-axis. Markov chains are the coloured lines in the middle of the trace plot. Markov chains can be deemed good-mixing and stationary if they oscillate round a central equilibrium. These two traits are a solid convergence indicator. 

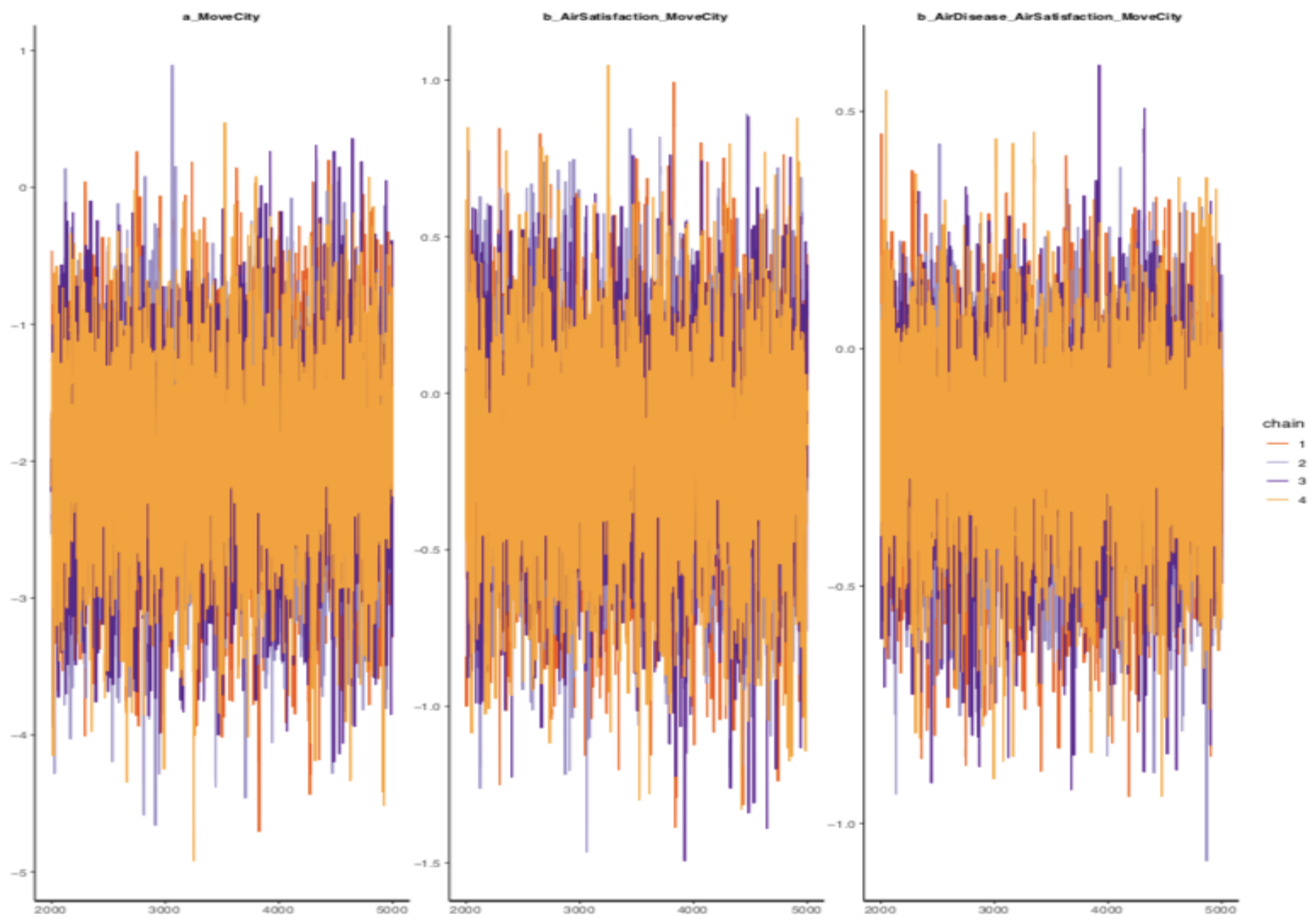

Figure 3: Trace plots for the posterior parameters of the Model

Figure 4 shows Gelman graphs of Model's parameters. The shrink factor (or Gelman factor) is used to measure the ratio between the variation between Markov chains and the variance within chains on the $y$-axis of the Gelman plot. Meanwhile, the x-axis depicts the simulation's iteration order. As can be seen, throughout the warm-up iterations, the shrink factors of all parameters rapidly drop to 1, indicating that there is no divergence across Markov chains. As a result, the Markov property is preserved. 
a_MoveCity

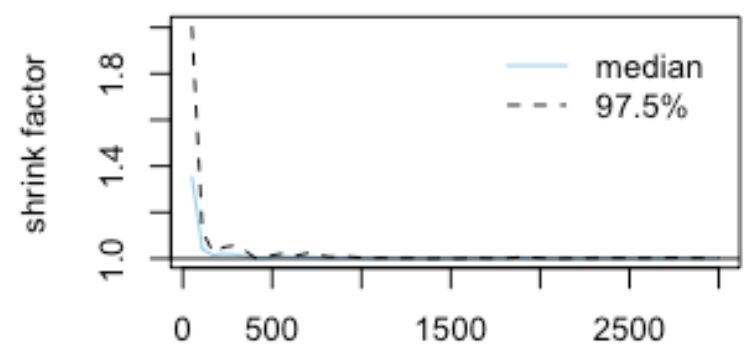

last iteration in chain
b_AirSatisfaction_MoveCity

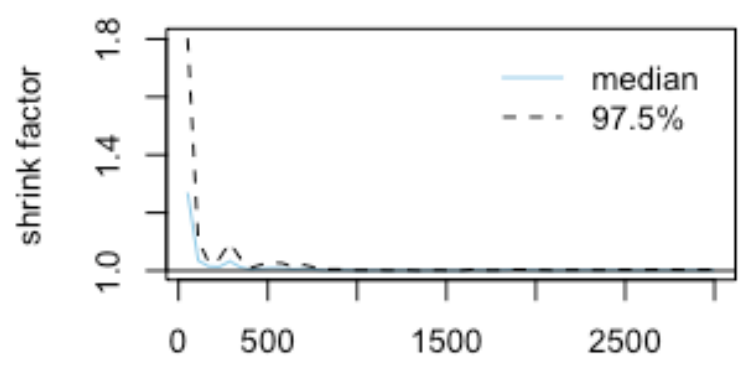

last iteration in chain

b_AirDisease_AirSatisfaction_MoveCity

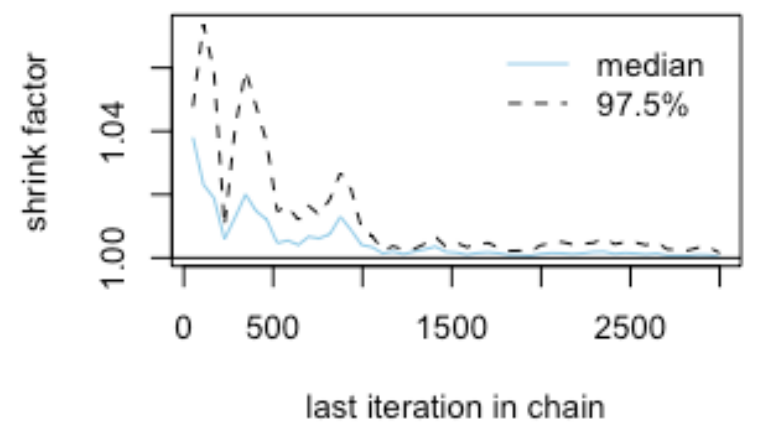

Figure 4: Gelman plots for Model's posterior parameters

Another further step to validate the convergence of Model's is to diagnose the Markov chains' autocorrelation levels visually (see Figure 5). The x-axes of the autocorrelation plots represents the number of Markov chains' lag, while the y-axes show the average level of autocorrelation of each chain. Visually, the average autocorrelation level declines substantially before the fifth lag, inducing all parameters to acquire a great number of effective samples. The autocorrelation plots' demonstrations again confirm the convergence of Model's Markov chains. 
a_MoveCity

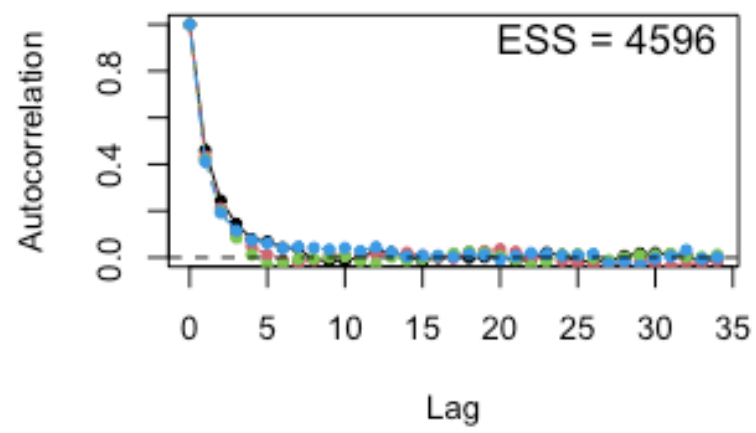

b_AirDisease_AirSatisfaction_MoveCity

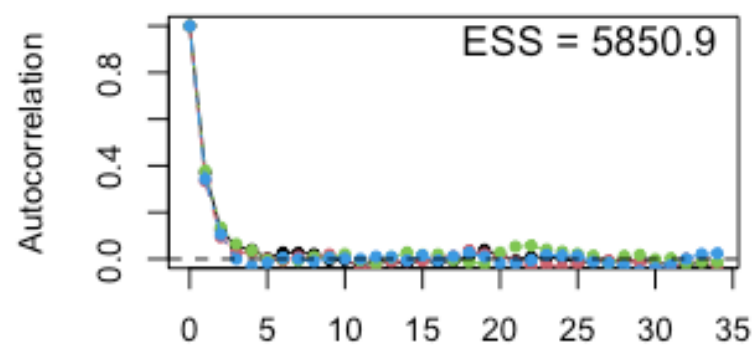

Lag
b_AirSatisfaction_MoveCity

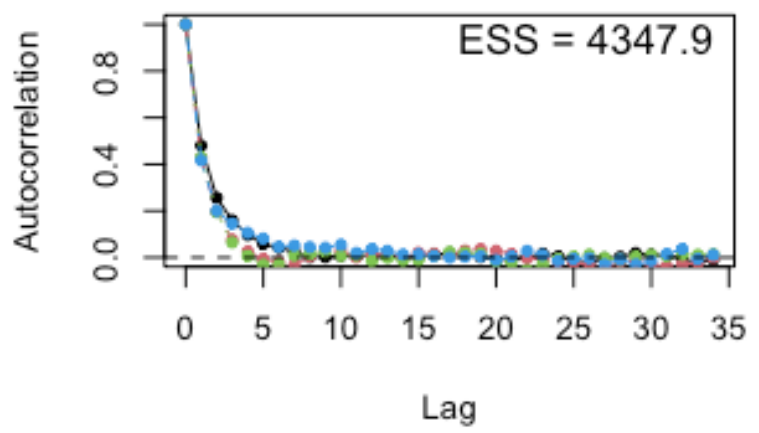

Figure 5: Autocorrelation plots for Model's posterior parameters

We discovered that people' satisfaction with air quality was negatively associated with their intention to relocate to another city ( $\mu_{\text {Airsatisfaction }}=-0.18$ and $\left.\sigma_{\text {Airsatisfaction }}=0.33\right)$, based on the simulated posterior results of Model. This finding supports our hypothesis that satisfied people are less inclined to migrate because the benefits of leaving the city are lower. The cost-benefit analysis of urban inhabitants' movement decisions as a result of air pollution, on the other hand, is far more complicated. Perceived a family member is affected by poor air quality moderated the effect of air satisfaction on migration intention $\left(\mu_{\text {Airsatisfaction } * \text { AirDisease }}=-0.21\right.$ and $\sigma_{\text {AirSatisfaction } * \text { AirDisease }}=0.20$ ). 

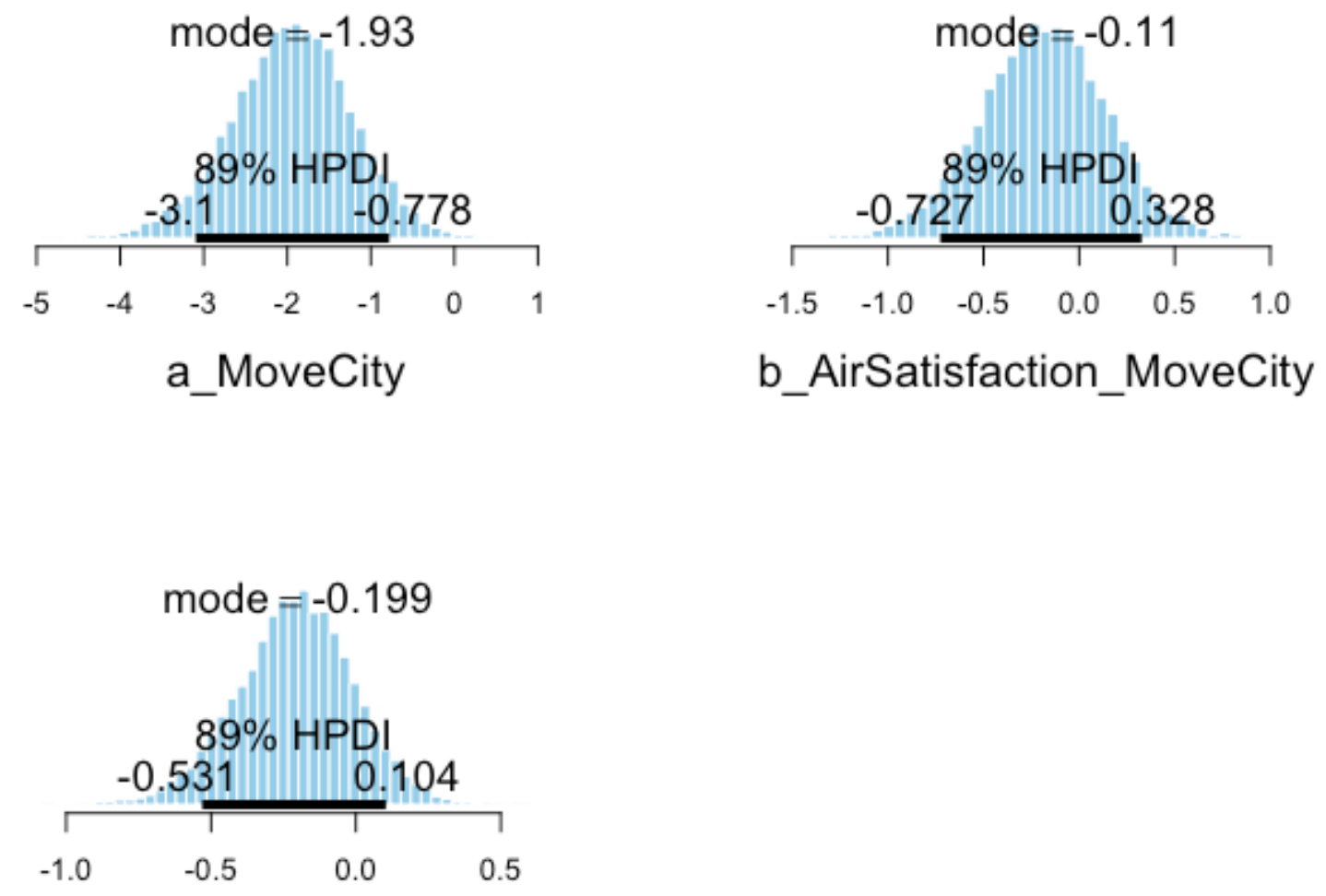

\section{b_AirDisease_AirSatisfaction_MoveCity}

Figure 6: Distributions Model's posterior coefficients with HPDI 89\%

The interval plot (see Figures 6) is used to test the dependability of Model's parameter distributions. The x-axis of the structure displays the posterior values of parameters. The coefficient of air satisfaction is mostly on the negative side of the axis, as can be seen in the graph, indicating a reliable negative relationship between AirSatisfaction and MoveCity. The distributions of the coefficients AirSatisfaction and AirSatisfaction*AirDisease are both negative, showing that the moderation effects of perceived availability of a better alternative and lower moving costs are most likely to be negative.

Note from figure 6 that AirSatisfaction*AirDisease has a greater moderation effect. Because the $\mathrm{x}$-axis has a higher proportion of negative values than the $\mathrm{y}$-axis, the moderating effect of AirSatisfaction can be considered more dependable than AirSatisfaction*AirDisease. 


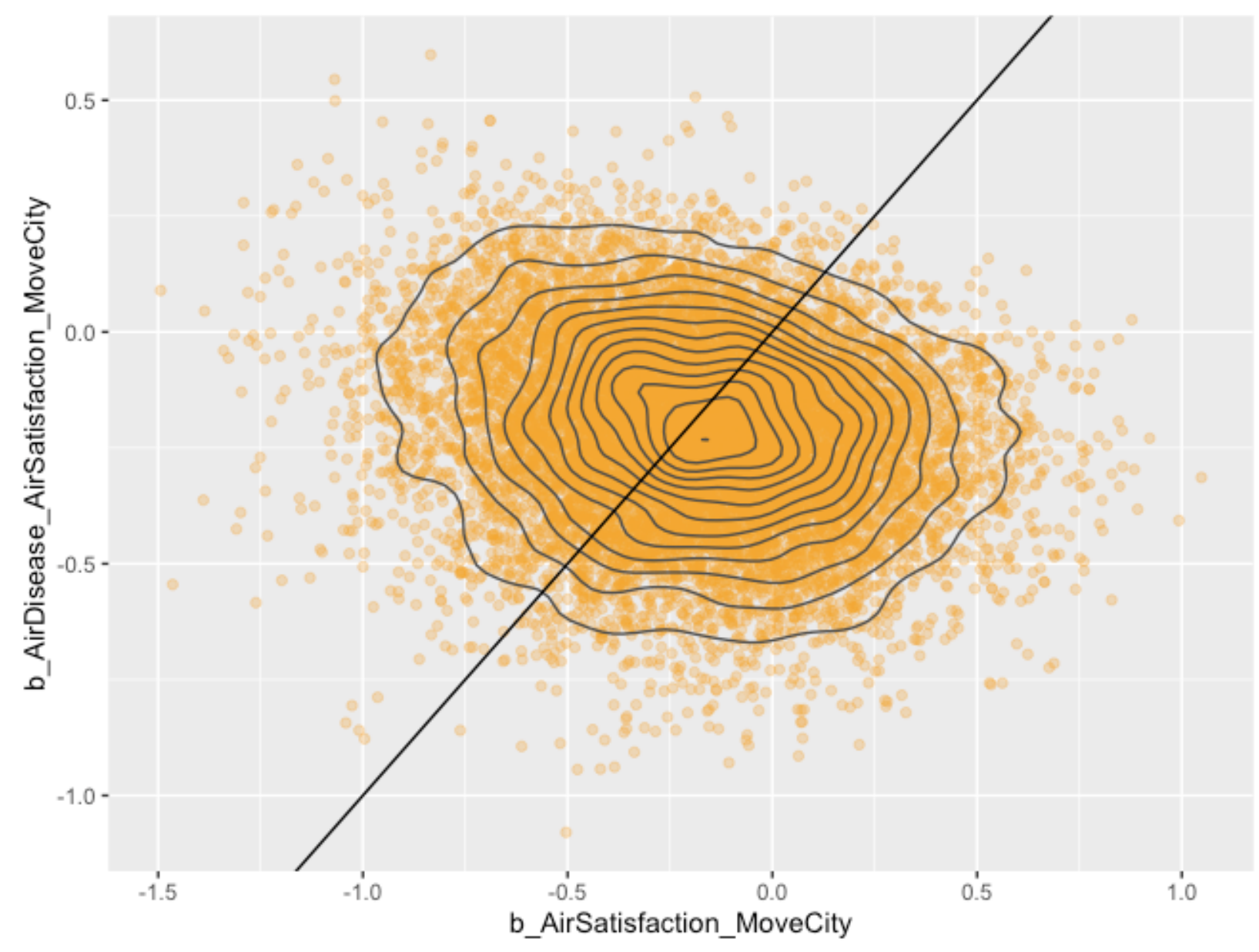

Figure 7: Pairwise distribution plot for Model 1's AirSatisfaction and AirSatisfaction*AirDisease

\section{Discussion}

Without a doubt, technological advancements make our world easier, and while it may appear difficult to prevent the adverse effects of air pollution, but it is the need of the hour. The paper's purpose is to investigate the effects of people's satisfaction with current air quality, as well as the moderating effects of air pollution on the health of their families. The data for this study came from two publicly available datasets on Hanoi people's views on air pollution. The majority of the samples came from people between the ages of 10 and 30 (it's $61.47 \%$ ). Nearly 5\% of the 475 respondents indicated they planned to migrate their family and employment to another city due to air pollution. We employed Bayesian analysis. The paper's aims to understand the psychological elements that govern human behaviour. The paper's purpose is to investigate the effects of people's satisfaction with current air quality, as well as the moderating effects of air pollution on the health of their families. People's satisfaction with air quality was negatively associated with their intent to move. But, if there are too many disadvantages to migration and it is viewed as financially draining, the notion to migrate is rejected. We found that families, where someone had already been a victim of a disease caused by air pollution, were more likely to move. Cost beneficial as medical treatment is very expensive. Health concerns to keep the family safe from other impending diseases. Increasing education, training, public awareness, and public participation are some of the 
key steps for optimizing the chances of meeting the targets and goals on the critical issue of climate change and pollution. Governments should disseminate appropriate information, educate citizens, and involve professionals in these concerns in order to successfully control the rise of the problem. Despite the challenge of eliminating human environmental pollution, a viable solution might be imagined as a close partnership between authorities, bodies, and physicians to bring the issue under control. As a supplement to the successful handling of the harmful health impacts associated with air pollution, a global preventative policy should be created to address human air pollution.

We argue based on our findings that environmental issues should not be restricted to the scope of the environmental study itself. Economics and sociology must be taken into account. The concepts of socio economics may seem insignificant bur environmental stressors can act as catalysts to mass migration, especially when low- and middle-income countries are more severely affected by such climate change hazards [23]. Aside from political interventions and technological infrastructure development [24], it is necessary to replace the current 'eco-deficit culture' among the developing economies with the 'eco-surplus culture,' particularly among private sectors, to prevent environmental problems from worsening $[25,26]$.

For the sake of transparency, we've listed the limitations of our research [27]. Because the samples were only taken in a city, the findings may not be indicative of the relationships between health and economic determinants and migration intentions among rural residents. Furthermore, because our interpretations were focused on Hanoi's environmental and economic development levels, the effects of health and economic determinants on migration should be confirmed using samples from other developing nations, not just Vietnam.

Acknowledgement

We specially thanks our instructors Abhijit Dasgupta and Anish Roychowdhury, professors in SP Jain School of Global Management, Australia, for helping us writing the manuscript. Besides, we would like to thanks Centre for Interdisciplinary Social Research, the research centre of Phenikaa University, Vietnam, for inspiring ideas and providing datasets to write the paper!

Supplementary

The code and dataset are available at: https://github.com/locquangnguyen8/AirPollution

Reference

[1] J. R. Newman, "Effects of industrial air pollution on wildlife," Biological Conservation, vol. 15, no. 3, pp. 181-190, 1979.

[2] N. Gouveia and T. Fletcher, "Time series analysis of air pollution and mortality: effects by cause, age and socioeconomic status," Journal of Epidemiology \& Community Health, vol. 54, no. 10, pp. 750-755, 2000.

[3] N. Künzli et al., "Public-health impact of outdoor and traffic-related air pollution: a European assessment," The Lancet, vol. 356, no. 9232, pp. 795-801, 2000.

[4] A. J. Cohen et al., "The global burden of disease due to outdoor air pollution," Journal of Toxicology and Environmental Health, Part A, vol. 68, no. 13-14, pp. 1301-1307, 2005.

[5] I. Air, "World Air Quality Report. Region \& City PM2. 5 Ranking. IQ Air, 2020," ed, 2019. 
[6] L. M. Luong, D. Phung, P. D. Sly, L. Morawska, and P. K. Thai, "The association between particulate air pollution and respiratory admissions among young children in Hanoi, Vietnam," Science of the total environment, vol. 578, pp. 249-255, 2017.

[7] Q.-H. Vuong, T.-T. Le, Q.-L. Nguyen, Q.-T. Nguyen, and M.-H. Nguyen, "Escaping from air pollution: The psychological process of domestic migration intention among urban people," arXiv preprint arXiv:2108.00497, 2021.

[8] Q.-H. Vuong, T.-T. Le, N. Quang-Loc, and M.-H. Nguyen, "Investigation into the rationale of migration intention due to air pollution integrating the Homo Oeconomicus traits," 2021.

[9] Q.-H. Vuong, T. V. Phu, T.-A. T. Le, and Q. Van Khuc, "Exploring Inner-City Residents' and Foreigners' Commitment to Improving Air Pollution: Evidence from a Field Survey in Hanoi, Vietnam," Data, vol. 6, no. 4, p. 39, 2021.

[10] Q. Van Khuc, T. V. Phu, and P. Luu, "Dataset on the Hanoian suburbanites' perception and mitigation strategies towards air pollution," Data in Brief, vol. 33, p. 106414, 2020.

[11] Q. H. Vuong and N. K. Napier, "Acculturation and global mindsponge: an emerging market perspective," International Journal of Intercultural Relations, vol. 49, pp. 354-367, 2015.

[12] Q. H. Vuong, "Global mindset as the integration of emerging socio-cultural values through mindsponge processes," Global Mindsets: Exploration and Perspectives, pp. 109-126, 2016.

[13] M.-H. Nguyen, T.-T. Le, M.-T. Ho, H. T. T. Nguyen, and Q.-H. Vuong, "Alice in suicideland: exploring the suicidal ideation mechanism through the sense of connectedness and help-seeking behaviors," International journal of environmental research and public health, vol. 18, no. 7, p. 3681, 2021.

[14] Q.-H. Vuong, M.-H. Nguyen, and T.-T. Le, "Home scholarly culture, book selection reason, and academic performance: Pathways to book reading interest among secondary school students," European Journal of Investigation in Health, Psychology and Education, vol. 11, no. 2, pp. 468-495, 2021.

[15] Q.-H. Vuong, M.-H. Nguyen, and T.-T. Le, A mindsponge-based investigation into the psycho-religious mechanism behind suicide attacks. Walter de Gruyter $\mathrm{GmbH}, 2021$.

[16] M. Baker, "Over half of psychology studies fail reproducibility test," Nature, 2015/08/27 2015, doi: https://doi.org/10.1038/nature.2015.18248.

[17] O. S. Collaboration, "Estimating the reproducibility of psychological science," (in en), Science, vol. 349, no. 6251, 2015/08/28/2015, doi: 10.1126/science.aac4716.

[18] K. Csilléry, M. G. B. Blum, O. E. Gaggiotti, and O. François, "Approximate Bayesian computation (ABC) in practice," Trends in ecology \& evolution, vol. 25, no. 7, pp. 410418, 2010 2010. [Online]. Available: files/1680/S0169534710000662.html.

[19] D. Kerkhoff and F. W. Nussbeck, "The influence of sample size on parameter estimates in three-level random-effects models," Frontiers in psychology, vol. 10, p. 1067, 2019.

[20] D. B. Dunson, "Commentary: practical advantages of Bayesian analysis of epidemiologic data," American journal of Epidemiology, vol. 153, no. 12, pp. 1222-1226, 2001.

[21] Q.-H. Vuong, V.-P. La, M.-H. Nguyen, M.-T. Ho, T. Tran, and M.-T. Ho, "Bayesian analysis for social data: A step-by-step protocol and interpretation," Methods $X$, vol. 7, p. 100924, 2020.

[22] Q.-H. Vuong, V.-P. La, M.-H. Nguyen, M.-T. Ho, M.-T. Ho, and P. Mantello, "Improving Bayesian statistics understanding in the age of Big Data with the bayesvl R package," Software Impacts, vol. 4, p. 100016, 2020. 
[23] S. Feng, A. B. Krueger, and M. Oppenheimer, "Linkages among climate change, crop yields and Mexico-US cross-border migration," Proceedings of the national academy of sciences, vol. 107, no. 32, pp. 14257-14262, 2010.

[24] Q.-H. Vuong, M.-T. Ho, H.-K. T. Nguyen, and M.-H. Nguyen, "The trilemma of sustainable industrial growth: Evidence from a piloting OECD's Green city," Palgrave Communications, vol. 5, no. 1, pp. 1-14, 2019.

[25] Q. H. Vuong, "Western monopoly of climate science is creating an eco-deficit culture," Economy, Land \& Climate Insight, 2021.

[26] Q. H. Vuong, V. P. La, H. K. T. Nguyen, M. T. Ho, T. T. Vuong, and M. T. Ho, "Identifying the moral-practical gaps in corporate social responsibility missions of Vietnamese firms: An event-based analysis of sustainability feasibility," Corporate Social Responsibility and Environmental Management, vol. 28, no. 1, pp. 30-41, 2021.

[27] Q.-H. Vuong, "Reform retractions to make them more transparent," Nature, vol. 582, no. $7811,2020$. 